\title{
Analysis of Full-Wave Conductor System Impedance over Substrate Using Novel Integration Techniques
}

\author{
Xin $\mathrm{Hu}$ \\ M.I.T. \\ xinhu@mit.edu \\ Jacob White \\ M.I.T. \\ white@mit.edu
}

\author{
Jung Hoon Lee \\ M.I.T. \\ junghoon@mit.edu \\ Luca Daniel \\ M.I.T. \\ luca@mit.edu
}

\begin{abstract}
An efficient approach to full-wave impedance extraction is developed that accounts for substrate effects through the use of two-layer media Green's functions in a mixed-potential-integral-equation (MPIE) solver. Particularly, the choice of implementation for the layered media Green's functions motivates the development of accelerated techniques for both volume and surface integrations in the solver. Solver accuracy is validated against measurements taken on fabricated devices; solver efficiency is demonstrated by its $9.8 \mathrm{X}$ reduction in cost in comparison to the traditional integration approach.
\end{abstract}

Categories and Subject Descriptors: B.7.2 [Design Aids]: Placement and routing, Simulation, Verification.

General Terms: Algorithms.

Keywords: Impedance extraction, Substrate modeling, Integral equation solver.

\section{INTRODUCTION}

The integration of RF, analog and digital circuitry on a single integrated-circuit substrate, or system-on-chip (SoC), has become a popular solution for various mixed-signal applications. However, due to the conductive nature of the substrate, it becomes an increasingly difficult task to ensure the correct operations of all the SoC components. The semiconducting silicon substrate used in most SoC systems permits noise injection and propagation, thereby exposing sensitive circuitry to substrate noise coupling. The severity of the substrate effects only worsens as operating frequencies increase. For instance, the induced eddy currents in the substrate may degrade the quality factor of high-frequency RF inductors, thus leading to poor analog performance. In addition to substrate losses, conductor skin and proximity effects may impact current return paths in a network of closely-spaced conductors. Moreover, electro-magnetic interference (EMI) in the SoC system may affect components through long-range propagation of electromagnetic waves. For example, the on-chip power and ground wires of

Permission to make digital or hard copies of all or part of this work for personal or classroom use is granted without fee provided that copies are not made or distributed for profit or commercial advantage and that copies bear this notice and the full citation on the first page. To copy otherwise, to republish, to post on servers or to redistribute to lists, requires prior specific permission and/or a fee.

DAC 2005, June 13-17, 2005, Anaheim, California, USA

Copyright 2005 ACM 1-59593-058-2/05/0006 ...\$5.00. lengths comparable to the wavelength could be potential sources of long-distance EMI emissions.

In order to avoid all the above problems in a circuit design, a simulation tool is needed that can accurately and efficiently extract full-wave conductor impedances in the presence of a conducting substrate. The solver described in this paper accomplishes just that. In the solver, the effects of the substrate are accounted for through the use of the well-developed complex image theory $[3,4,5,6]$, which generates a set of closed-form, full-wave, 3D layered vector and scalar Green's functions for a two-layer medium. These layered media Green's functions are then incorporated into a MPIE formulation to compute the $3 \mathrm{D}$ vector and scalar potentials for the conductors. Since the substrate effects are already captured by the layered media Green's functions, the MPIE formulation performs 3D conductor impedance extraction without the need to discretize the substrate's volume or surface. In the MPIE formulation, the choice of the closed-form, full-wave layered media Green's function kernels motivates the development in this paper of a set of novel, accelerated volume and surface integration schemes. These schemes reduce the $3 \mathrm{D}$ volume or $2 \mathrm{D}$ surface integrations of the layered media Green's function kernels to a sum of 1D line integrals. These techniques dramatically improve the efficiency of the solver, thus making it a viable solution to full-wave substrate impedance extractions of complex interconnect structures.

Many other numerical techniques have been proposed to analyze substrate effects on conductor networks. In [16, 13, 10, 14], 2D quasistatic layered media Green's functions are used to model substrate impedance. In [12], a quasi-magnetostatic integral formulation is applied to both the conductors and the substrate. In [15], a CAD-oriented modeling approach is used that simulates substrate effects by ideal lumped circuit elements. In [8], vector conductor potentials are approximated using a zero-thickness formulation, and substrate effects are accounted for through full-wave layered media Green's functions. Due to the numerous approximations made, these methods lack accuracy. In [11, 9], full-wave substrate modeling is accomplished based on applying computationally expensive finite-element and finite-difference time-domain methods to a finely-discretized substrate.

Overall, the solver described in this paper is novel in that it possesses a unique combination of full-wave layered media Green's functions (Section 2), a method for 3D conductor vector and scalar potential computations (Section 3), and accelerated techniques for both volume and surface integrations of the full-wave kernels (Section 4). Finally, examples are presented that validate the accuracy and efficiency of the solver against measurements made on fabricated devices (Section 5). 


\section{BACKGROUND}

\subsection{MPIE Formulation}

As in $[17,18]$, the following set of integral equations can be used to compute conductor current distribution $\bar{J}$ and conductor surface charge $\rho$,

$$
\begin{aligned}
\frac{\bar{J}(r)}{\sigma}+j \omega \frac{\mu}{4 \pi} \int_{V} \bar{J}\left(r^{\prime}\right) G_{A}\left(r, r^{\prime}\right) d r^{\prime} & =-\nabla \phi(r) \\
\frac{1}{4 \pi \varepsilon} \int_{S} \rho\left(r^{\prime}\right) G_{\phi}\left(r, r^{\prime}\right) d r^{\prime} & =\phi(r) ; \\
\nabla \cdot \bar{J}(r) & =0 \\
\hat{n} \cdot \bar{J}(r) & =j \omega \rho(r),
\end{aligned}
$$

where $\mathrm{V}$ and $\mathrm{S}$ are the union of conductor volumes and surfaces respectively, $G_{A}$ and $G_{\phi}$ are the vector and scalar Green's functions respectively, $\phi$ is the scalar potential on the conductor surfaces, $\mu$ is the magnetic permeability, $\varepsilon$ is the dielectric constant, $\sigma$ is the conductivity, and $\omega$ is the angular frequency of the conductor excitation. For a given interconnect structure, after specifying a set of terminal voltages as inputs, terminal currents can be obtained by solving the above system of equations (1)-(4). Consequently, The overall system impedance for the structure can be extracted.

\subsection{Layered Media Green's Functions}

The layered media Green's functions adopted in this paper are based on the well-established complex image theory $[3,6,4]$. A planar-layered medium is shown in Fig. 1a. The source, either a vertical electrical dipole (VED) or a horizontal electric dipole (HED), is located at a height $h$ in the top-most layer. The location of the observation point, $z$, is also confined to the top-most layer. The radial distance parallel to the planar medium between the source dipole and the observation point is denoted by $d$. The $i$ th layer is characterized by its dielectric property, $\varepsilon_{i}=\varepsilon_{r_{i}}-j \frac{\sigma}{\varepsilon_{0} \omega}$, where $\varepsilon_{r_{i}}$ is the relative permittivity, and $\varepsilon_{0}$ is the free-space permittivity.

According to [1], in a multi-layered structure, a VED generates vector potentials only in the vertical z-direction $\left(G_{z z}^{A}\right)$, an x-directed HED generates vector potentials in both the $\mathrm{x}$-direction $\left(G_{x x}^{A}\right)$ and the z-direction $\left(G_{x z}^{A}\right)$, and an y-directed HED generates vector potentials in both the y-direction $\left(G_{y y}^{A}\right)$ and the z-direction $\left(G_{y z}^{A}\right)$. As for scalar potentials, different types of scalar potentials are associated with different orientations of the source dipoles, $G_{v}^{q}$ for a VED and $G_{h}^{q}$ for a HED.

These layered media Green's functions do not generally exist in closed form in the spatial domain. To facilitate their evaluation, the aforementioned Green's functions for the top-most layer are derived analytically in the spectral domain using a recursive algorithm. The complete set of closed-form spectral Green's functions can be found in [4].

To obtain the spatial counterparts to the spectral Green's functions, direct evaluation of their inverse transforms can be used, but the resulting expressions are highly oscillatory, thus are computationally expensive to evaluate [5]. Hence an approach is taken where the spectral Green's functions are approximated so as to generate closed forms in the spatial domain. Each spectral Green's function is approximated through its decomposition into two contributions:

1. Complex quasistatic term denoted by $c$

2. A sum of complex exponentials approximated using a twolevel Generalized Pencil-of-Function (GPOF) method [5, 6]. The exponentials are in the form of $\sum a_{i} e^{-k_{z} b_{i}}, j k_{x} \sum a_{i} e^{-k_{z} b_{i}}$ or $j k_{y} \sum a_{i} e^{-k_{z} b_{i}}$, where $a_{i}^{\prime} s$ and $b_{i}^{\prime} s$ are the complex coefficients and exponents, respectively, and $k_{z}$ is the wave number in the zdirection for the top-most layer.
Using the Sommerfeld Identity [21], the approximated spectral Green's functions can be easily transformed into the spatial domain where they have closed-forms. Table 1 lists the various spectral-to-spatial domain mappings utilized in the transformation process. In addi-

\begin{tabular}{|l|l|c|}
\hline $\begin{array}{l}\text { Image } \\
\text { Type }\end{array}$ & Spectral Domain & Spatial Domain \\
\hline real & $\tilde{G}=\mathrm{c}$ & $\begin{array}{c}G_{r i}(d, h, z)=c \frac{e^{-j k R}}{R}, \\
\mathrm{R}=\sqrt{d^{2}+(z+h)^{2}}\end{array}$ \\
\hline complex I & $\tilde{G}=\sum a_{i} e^{-k_{z} b_{i}}$ & $G_{c i 1}(d, h, z)=\sum a_{i} \frac{e^{-j k R}}{R}$, \\
& & $\mathrm{R}=\sqrt{d^{2}+\left(z+h-j b_{i}\right)^{2}}$ \\
\hline complex II & $\tilde{G}=-j k_{x} \sum a_{i} e^{-k_{z} b_{i}}$ & $G_{c i 2 x}(d, h, z)=\sum a_{i} \frac{\partial}{\partial x}\left(\frac{e^{-j k R}}{R}\right)$, \\
& & $\mathrm{R}=\sqrt{d^{2}+\left(z+h-j b_{i}\right)^{2}}$ \\
& $\tilde{G}=-j k_{y} \sum a_{i} e^{-k_{z} b_{i}}$ & $G_{c i 2 y}(d, h, z)=\sum a_{i} \frac{\partial}{\partial y}\left(\frac{e^{-j k R}}{R}\right)$, \\
& & $\mathrm{R}=\sqrt{d^{2}+\left(z+h-j b_{i}\right)^{2}}$ \\
\hline
\end{tabular}

Table 1: Table of spectral-to-spatial domain mappings.

tion, some of the spatial-domain layered media Green's functions require the contribution of the free-space Green's function with the form

$$
G_{f s}=\frac{e^{-j k R}}{R} \quad R=\sqrt{d^{2}+(h-z)^{2}},
$$

where $k=\omega \sqrt{\mu_{0} \varepsilon_{1}}$ is the magnitude of the wave number of the top-most layer.

All spatial-domain layered media Green's functions can be approximated by various combinations of $G_{f s}$, real images, and complex images [3]. More specifically, $G_{x x}^{A}, G_{y y}^{A}, G_{z z}^{A}, G_{v}^{q}$, and $G_{h}^{q}$ are expressed as some combinations of $G_{f s}$, a real image and type I complex images, while $G_{x z}^{A}$ and $G_{y z}^{A}$ contain only type II complex images.

The idea of the complex image theory becomes clear: the potential generated by a source dipole evaluated at an observation point in the presence of a layered medium is the same as the sum of potentials generated, in the absence of the layered medium, by some combination of the source dipole and image dipoles with real and complex locations in space. Fig. 1b illustrates this concept for $G_{x x}^{A}$ [2].

\section{INTEGRATION OF LAYERED MEDIA GREEN'S FUNCTIONS IN MPIE SOLVER}

The layered media vector and scalar Green's functions,

$$
G_{A}=\left[\begin{array}{ccc}
G_{x x}^{A} & 0 & 0 \\
0 & G_{y y}^{A} & 0 \\
G_{x z}^{A} & G_{y z}^{A} & G_{z z}^{A}
\end{array}\right], \quad G_{\phi}=\left\{\begin{array}{cc}
G_{v}^{q} & V E D \\
G_{h}^{q} & H E D
\end{array}\right.
$$

are substituted into (1) and (2), respectively, to account for the substrate effects on the vector and scalar potentials.

To solve (1)-(4) numerically, one approximates the conductor volume current and surface charge by a finite set of piece-wise constant basis functions. This means that the conductor volumes are discretized into short and thin filaments along their length and cross-sections with the assumption that a constant current density flows length-wise through each filament in the direction $\hat{l}=\left[l_{x}, l_{y}, l_{z}\right]$. Each filament has a length $d$ and cross-sectional area $A$. Likewise, the conductor surfaces are discretized into small panels with the assumption that charge density is constant on each panel. Each panel has an area $S$ and a centroid location $r_{c}$.

After discretizing (1) and (2), one applies a Galerkin technique [21] to (1) and a centroid collocation technique [18] to (2) to produce the 
following branch equations:

$$
\left[\begin{array}{cc}
R+j \omega L & 0 \\
0 & P
\end{array}\right]\left[\begin{array}{l}
I \\
q
\end{array}\right]=\left[\begin{array}{l}
V \\
\phi
\end{array}\right]
$$

where

$$
\begin{aligned}
R_{j j} & =\frac{1}{\sigma} \frac{d_{j}}{A_{j}} \\
L_{i j} & =\frac{\mu}{4 \pi} \int_{V_{i}} \int_{V_{j}} G_{A}\left(r, r^{\prime}\right) \hat{l}_{j} \cdot \hat{l}_{i} d r^{\prime} d r \\
P_{i j} & =\frac{A_{i}}{4 \pi \varepsilon} \int_{S_{j}} G_{\phi}\left(r_{c_{i}}, r^{\prime}\right) d r^{\prime} .
\end{aligned}
$$

Substituting (6.a) into (9) and (6.b) into (10) produces the following expanded forms:

$$
\begin{aligned}
L_{i j}= & \frac{\mu}{4 \pi} \int_{V_{i}} \int_{V_{j}}\left[G_{x x}^{A}\left(r, r^{\prime}\right) l_{x j} l_{x i}+G_{y y}^{A}\left(r, r^{\prime}\right) l_{y j} l_{y i}+G_{z z}^{A}\left(r, r^{\prime}\right) l_{z j} l_{z i}\right. \\
& \left.+G_{x z}^{A}\left(r, r^{\prime}\right) l_{x j} l_{z i}+G_{y z}^{A}\left(r, r^{\prime}\right) l_{y j} l_{z i}\right] d r^{\prime} d r \\
P_{i j}= & \frac{A_{i}}{4 \pi \varepsilon} \int_{S_{j}} G_{v / h}^{q}\left(r_{c_{i}}, r^{\prime}\right) d r^{\prime} .
\end{aligned}
$$

Since the layered media Green's functions are expressed as combinations of the closed forms shown in table 1 and (5), the integrations of (11) and (12) can be performed numerically involving only these closed forms.

The implication of (11) is that the potential at an evaluation filament due to the potential generated by a source filament in the presence of a layered substrate is the same as the potential generated, in the absence of the substrate, by a combination of filament sources with real and complex locations in space. This idea is illustrated in Fig. 1c. Similar concept also applies to scalar potential analysis in terms of panel charges.

Finally, equations (3) and (4) can be imposed on the discretized system defined by (7) using either nodal analysis [17] or mesh analysis [18], or a combination of both [19].

\section{NOVEL APPROACH TO INTEGRATION}

The choice of closed-form vector layered media Green's functions in (11) suggests that, for vector potential analysis, one only needs to be concerned with the double volume integration of two types of kernels:

$$
\begin{array}{cc}
\text { Type I: } & \frac{e^{-j k\left|r^{\prime}-r\right|}}{\left|r^{\prime}-r\right|} \\
\text { Type II: } \quad \frac{\partial}{\partial h}\left(\frac{e^{-j k\left|r^{\prime}-r\right|}}{\left|r^{\prime}-r\right|}\right) ; \quad \mathrm{h}=\mathrm{x} \text { or } \mathrm{y},
\end{array}
$$

where $r^{\prime}=\left[x^{\prime}, y^{\prime}, z^{\prime}\right]$ is a vector from the origin to the source point, and

$$
r= \begin{cases}{[x, y, z]} & \text { for } G_{f s} \\ {[x, y,-z]} & \text { for a real image } \\ {[x, y,-z+j b]} & \text { for a complex image }\end{cases}
$$

is a vector from the origin to the observation point or its image. As for scalar potential analysis, the use of the scalar layered media Green's functions in (12) suggests that only Type I kernels are needed for surface panel integrations.

\subsection{Volume Integration of Type I kernel}

As a consequence of using the Galerkin technique for vector potential computation, the resulting double volume integrals are extremely expensive to compute if a traditional 3D quadrature approach is applied to both inner and outer integrations. This observation motivates the development of a set of accelerated integration

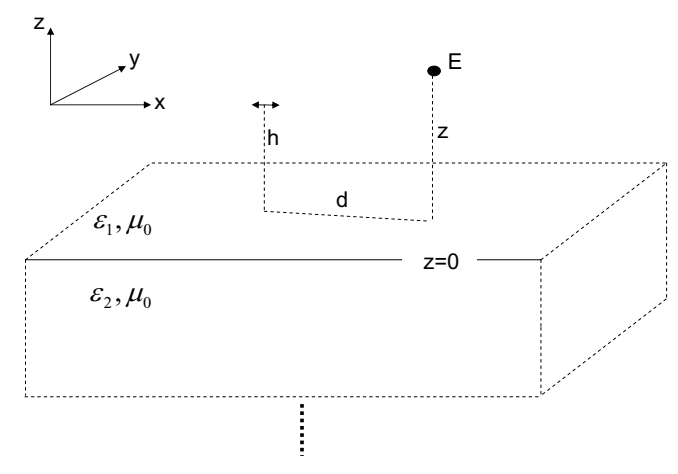

(a)
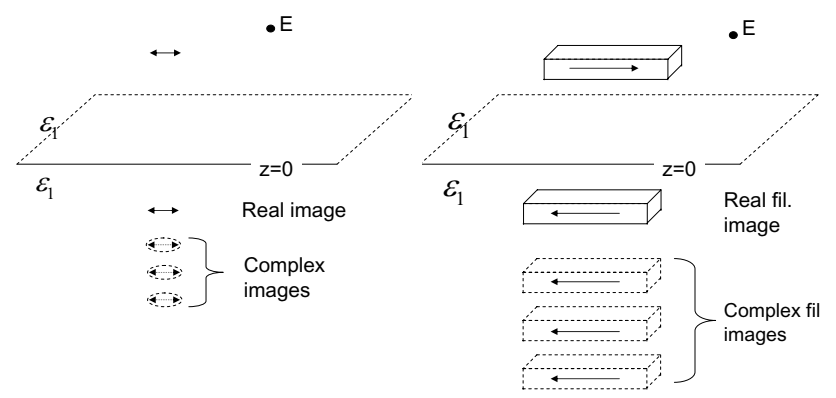

(b)

(c)

Figure 1: a. A source dipole and an evaluation point in a twolayered planar medium; $b$. Complex image representation of $G_{x x}^{A}$; c. Complex image representation of the fields generated by an $x$-directed source filament in the presence of a layered substrate.

schemes applicable to inner volume integrations, which when combined with a 3D quadrature approach to outer integrations, dramatically enhances the efficiency of the overall double volume integrations.

First, let's examine the case of

$$
\int_{V} \frac{e^{-j k\left|r^{\prime}-r\right|}}{\left|r^{\prime}-r\right|} d v^{\prime}
$$

Consider the distribution of sources within a filament of volume $V$. As shown in Fig. 2, the surface of $V$, denoted by $\partial V$, is composed of several faces, each indexed as $\partial_{j} V . \partial_{j} V$ has an outward unit normal $\hat{n}_{j}$. The perpendicular distance generated from the projection of the observation point $r$ onto the plane of $\partial_{j} V$ is denoted as $h_{j}$. This style of notation is adopted from [7].

The first step in evaluating the volume integral in (14) is to apply the Divergence theorem to transform the integral in $V$ to an integration over $\partial V$. The theorem states,

$$
\int_{V}(\nabla \cdot F) d v=\int_{\partial V} F \cdot \hat{n} d a
$$

Observation I: According to (15), the volume integration in (14) can be transformed to an integral over the volume's enclosing surfaces if a vector can be determined such that its divergence is the same as the integrand in (14).

In order to apply such theorem, the integration kernel must be continuously differentiable over the domain of integration $V$. This is not the case, however, when the observation point $r$ is in $\mathrm{V}$ or on 
$\partial V$. A separate treatment is devised to handle this self-term case as shown in the latter part of this section.

However, if $r$ is outside of $V$, the Divergence theorem is applied to (14) which yields

$$
\begin{aligned}
\int_{V} \frac{e^{-j k R}}{R} d v^{\prime} & =\int_{V} \nabla^{\prime} \cdot \vec{R}\left[\frac{1}{-j k} \frac{e^{-j k R}}{R^{2}}-\frac{1}{(-j k)^{2}}\left(\frac{e^{-j k R}}{R^{3}}-\frac{1}{R^{3}}\right)\right] d v^{\prime} \\
& =\int_{\partial V} \hat{n} \cdot \vec{R}\left[\frac{1}{-j k} \frac{e^{-j k R}}{R^{2}}-\frac{1}{(-j k)^{2}}\left(\frac{e^{-j k R}}{R^{3}}-\frac{1}{R^{3}}\right)\right] d s^{\prime} \\
& =\sum_{j} \int_{\partial_{j} V} \hat{n}_{j} \cdot \vec{R}\left[\frac{1}{-j k} \frac{e^{-j k R}}{R^{2}}-\frac{1}{(-j k)^{2}}\left(\frac{e^{-j k R}}{R^{3}}-\frac{1}{R^{3}}\right)\right] d s^{\prime} \\
& =\sum_{j} h_{j} \int_{\partial_{j} V}\left[\frac{1}{-j k} \frac{e^{-j k R}}{R^{2}}-\frac{1}{(-j k)^{2}}\left(\frac{e^{-j k R}}{R^{3}}-\frac{1}{R^{3}}\right)\right] d s^{\prime},
\end{aligned}
$$

where $\vec{R}$ is a vector from $r$ to $r^{\prime}$, and $R=\|\vec{R}\|$.

In (16), the 3D integration in $V$ is transformed to a sum of $2 \mathrm{D}$ integrations over faces bounding V. In order to proceed further, more properties have to be defined for $\partial_{j} V$ in relation to the observation point $r$ as shown in Fig. 2:

- $P$ is the end point of the projection of $r$ onto the plane defined by $\partial_{j} V$.

- $\partial_{j} V$ is enclosed by a set of edges, each indexed as $\partial_{i} \partial_{j} V$.

- For each $\partial_{i} \partial_{j} V$, a local coordinate system is defined with the origin centered at $P$ and three axes of directions, $\hat{l}_{i j}, \hat{u}_{i j}$ and $\hat{n}_{j}$.

- $d_{i j}$ is the perpendicular distance of the projection of $\mathrm{P}$ onto the line $\partial_{i} \partial_{j} V$.

- $\vec{\rho}$ is a vector from $P$ to a point on $\partial \partial_{j} V$, the boundary of $\partial_{j} V$, and $\rho$ denotes the distance between the two points.

Observation II: The Divergence theorem can be applied once more to transform integration over surface $\partial_{j} V$ to one over contour $\partial_{j} V$ of $\partial_{j} V$.

However, if $P$ is in $\partial_{j} V$ or on $\partial \partial_{j} V$, before such theorem can be applied, it is necessary to exclude for separate treatment a region of $\partial_{j} V_{\varepsilon}$, which is the intersection of $\partial_{j} V$ and a small disk of radius $\varepsilon$ centered at $P$. Let $I(R)$ be the integrand in (16) such that

$$
\begin{aligned}
& \int_{V} \frac{e^{-j k R}}{R} d v^{\prime}=\sum_{j} h_{j} \int_{\partial_{j} V} I(R) d s^{\prime} \\
& =\lim _{\varepsilon \rightarrow 0} \sum_{j} h_{j} \int_{\partial_{j}\left(V-V_{\varepsilon}\right)} I(R) d s^{\prime}+\lim _{\varepsilon \rightarrow 0} \sum_{j} h_{j} \int_{V_{\varepsilon}} I(R) d s^{\prime} \\
& \quad=\lim _{\varepsilon \rightarrow 0} \sum_{j} h_{j} \int_{\partial_{j}\left(V-V_{\varepsilon}\right)} \nabla^{\prime} \cdot \frac{\vec{\rho}}{\rho^{2}}\left[\frac{1}{(-j k)^{2}}\left(\frac{e^{-j k R}}{R}-\frac{1}{R}\right)-\frac{1}{-j k}\right] d s^{\prime} \\
& =\lim _{\varepsilon \rightarrow 0} \sum_{j} h_{j} \int_{\partial_{j}\left(V-V_{\varepsilon}\right)} \hat{u} \cdot \frac{\vec{\rho}}{\rho^{2}}\left[\frac{1}{(-j k)^{2}}\left(\frac{e^{-j k R}}{R}-\frac{1}{R}\right)-\frac{1}{-j k}\right] d l^{\prime} \\
& =\sum_{j} h_{j} \sum_{i} \int_{\partial_{i} \partial_{j} V} \frac{d_{i j}}{\rho^{2}}\left[\frac{1}{(-j k)^{2}}\left(\frac{e^{-j k R}}{R}-\frac{1}{R}\right)-\frac{1}{-j k}\right] d l^{\prime} \\
& \quad+\lim _{\varepsilon \rightarrow 0} \sum_{j} h_{j} \int_{\partial_{j} V_{\varepsilon}} \hat{u} \cdot \frac{\vec{\rho}}{\rho^{2}}\left[\frac{1}{(-j k)^{2}}\left(\frac{e^{-j k R}}{R}-\frac{1}{R}\right)-\frac{1}{-j k}\right] d l^{\prime},
\end{aligned}
$$

where $\hat{u}$ is the outward normal vector of $\partial_{j} V \backslash \partial_{j} V_{\varepsilon}$.
The second integral of (17) can be evaluated using a local polar coordinate system centered at $P$. This produces:

$$
\begin{aligned}
& \lim _{\varepsilon \rightarrow 0} \sum_{j} h_{j} \int_{\partial \partial_{j} V_{\varepsilon}} \hat{u} \cdot \frac{\vec{\rho}}{\rho^{2}}\left[\frac{1}{(-j k)^{2}}\left(\frac{e^{-j k R}}{R}-\frac{1}{R}\right)-\frac{1}{-j k}\right] d l^{\prime} \\
& =\sum_{j} h_{j} \sum_{i} \int_{\partial_{i} \partial_{j} V} \frac{d_{i j}}{\rho^{2}}\left[\frac{1}{(-j k)^{2}}\left(\frac{e^{-j k|h|}}{|h|}-\frac{1}{|h|}\right)-\frac{1}{-j k}\right] d l^{\prime} .
\end{aligned}
$$

Substituting (18) into (17) yields the final expression

$\int_{V} \frac{e^{-j k R}}{R} d v^{\prime}=\sum_{j} h_{j} \sum_{i} \int_{\partial_{i} \partial_{j} V} \frac{d_{i j}}{\rho^{2}}\left[\frac{1}{(-j k)^{2}}\left(\frac{e^{-j k R}-1}{R}-\frac{e^{-j k|h|}-1}{|h|}\right)\right]_{(19)} d l^{\prime}$.

Equation (19) shows that the volume integration of (14) can be transformed to a sum of 1D integrations over the line segments contouring the volume.

\subsubsection{Self-term Volume Integration}

For the separate case where $r \in V \backslash \partial V$, a simple approximation scheme is used. That is,

$$
\int_{V} \frac{e^{-j k\left|r-r^{\prime}\right|}}{\left|r-r^{\prime}\right|} d v^{\prime}=\sum_{i} e^{-j k\left|r-r_{i c}\right|} \int_{V_{i}} \frac{1}{\left|r-r^{\prime}\right|} d r^{\prime},
$$

where the source filament is divided into sub-filaments, and $r_{i c}$ is the center of the ith sub-filament. The integral in (20) is solved using the method described in [7] which transforms the volume integral of $\frac{1}{\left|r-r^{\prime}\right|}$ into a sum of boundary segment integrals through the application of the Divergence theorem. The effect of extracting the $e^{-j k R}$ term outside of the integral is minimal since both source and evaluation points vary within only one filament-length, which is a small fraction of the wavelength.

\subsection{Volume Integration of Type II kernel and Surface Integration of Type I kernel}

Let's examine the case of

$$
\int_{V} \frac{\partial}{\partial h} \frac{e^{-j k\left|r^{\prime}-r\right|}}{\left|r^{\prime}-r\right|} d v^{\prime}, \text { where } h=x \text { or } y .
$$

Since only $G_{x z}^{A}$ and $G_{y z}^{A}$ require the use of Type II kernels, there is no need to be concerned with the self-term case where $r \in V \backslash \partial V$.

Observation III: Through two consecutive applications of the Divergence theorem, the volume integral of (21) can also be transformed to a sum of 1D line integrals. That is,

$$
\begin{aligned}
\int_{V} \frac{\partial}{\partial h} \frac{e^{-j k\left|r^{\prime}-r\right|}}{\left|r^{\prime}-r\right|} d v^{\prime} & =-\int_{V} \nabla^{\prime} \cdot\left(\hat{h} \frac{e^{-j k R}}{R}\right) d v^{\prime} \\
& =-\sum_{j} n_{j_{h}} \int_{\partial_{j} V} \frac{e^{-j k R}}{R} d s^{\prime} \\
& =-\sum_{j} n_{j_{h}} \int_{\partial_{j} V} \nabla^{\prime} \cdot \frac{\vec{\rho}}{\rho^{2}}\left(\frac{e^{-j k R}}{-j k}-\frac{1}{-j k}\right) d s^{\prime} \\
& =-\sum_{j} n_{j_{h}} \sum_{i} \frac{d_{i j}}{\rho^{2}} \int_{\partial_{i} \partial_{j} V}\left(\frac{e^{-j k R}}{-j k}-\frac{1}{-j k}\right) d l^{\prime}
\end{aligned}
$$

where $n_{j_{h}}$ is either the x or y component of $\hat{n}_{j}$.

Observation IV: To determine the surface integration of the Type I kernel, $\int_{S} \frac{e^{-j k\left|r-r^{\prime}\right|}}{\left|r-r^{\prime}\right|} d s^{\prime}$, one immediately sees that the solution is already obtained in (22) where the integrand generated after applying the first Divergence theorem is exactly the same as the integrand of the surface integration. 


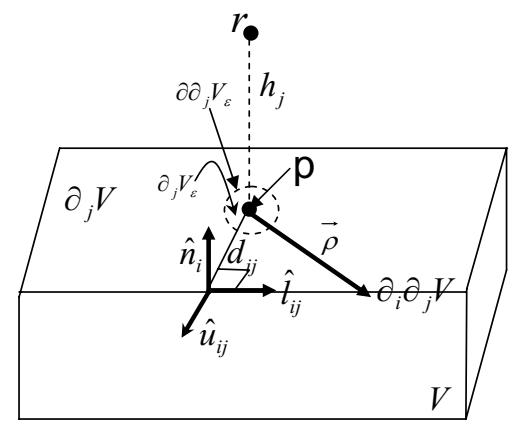

Figure 2: Geometric definition for quantities used in the integration schemes.

\section{RESULTS}

The purpose of the first example in this section is to establish the efficiency of the solver due its use of the accelerated integration schemes. The purpose of the second example is to validate the simulation techniques introduced in this paper by comparing measurement data obtained for a fabricated RF inductor to the results produced from simulations. The third example demonstrates the solver's ability to capture conductive substrate effects at the integrated-circuit level. The final example verifies the solver's ability to capture full-wave effects on conductor impedance in the presence of a substrate.

\subsection{Computational Cost Comparison}

This section presents cost analysis and comparison of utilizing the accelerated integration scheme as opposed to a standard 3D Gaussian quadrature approach for the volume filament integration of a Type I kernel. The source filament is $1 \mathrm{um} \times 1 \mathrm{um}$ in the crosssection and 500um in length. The potential is evaluated $2.5 \mathrm{um}$ from the center of the filament. Table 2 shows that, in comparison to

\begin{tabular}{|c|c|c|c|}
\hline $\begin{array}{c}\text { Accuracy } \\
(\%)\end{array}$ & $\begin{array}{c}\text { 3D Gaussian } \\
\text { quadrature (pts \#) }\end{array}$ & $\begin{array}{c}\text { 1D accelerated } \\
\text { method (pts \#) }\end{array}$ & $\begin{array}{c}\text { cost } \\
\text { reduction }\end{array}$ \\
\hline 0.1 & 1755 & 240 & $7.5 \mathrm{X}$ \\
\hline 1 & 1062 & 132 & $8 \mathrm{X}$ \\
\hline 4 & 675 & 48 & $14 \mathrm{X}$ \\
\hline
\end{tabular}

Table 2: Cost analysis for the volume integration of a Type I kernel.

the standard 3D Gaussian quadrature scheme, an average of $9.8 \mathrm{X}$ reduction in computational cost is achieved when the accelerated integration scheme is used for close-field potential calculations.

\subsection{Accuracy Validation Against Measurements}

Fig. 3 shows a plot of quality factors (Q-factors) obtained for a square 2.75-turn spiral RF inductor excited over a range of frequency inputs. One set of Q-factor data is produced from measurements while the other set is from the solver introduced in this paper.

The RF inductor, as shown in Fig. 4a, is fabricated on a multichip module (MCM) [20] using copper wires that are $5 \mu \mathrm{m}$ thick and $68.75 \mu \mathrm{m}$ wide. The separation distance between the turns is $25 \mu \mathrm{m}$. The overall area of the inductor is $1 \mathrm{~mm}^{2}$. A guard ring is placed $200 \mu \mathrm{m}$ from the RF-inductor to simulate a nearby ground. This structure is embedded in a dielectric and situated $150 \mu \mathrm{m}$ above the substrate. The dielectric has a resistivity $(\rho)$ of $1.0 \times 10^{17} \Omega \cdot \mathrm{cm}$ and a relative permittivity $\left(\varepsilon_{r}\right)$ of 3.5 . For the substrate, $\rho=1.0 \times 10^{14} \Omega$. $\mathrm{cm}$ and $\varepsilon_{r}=9.9$.

Measuring Q-factors of fabricated RF inductors is a difficult task, and measurements are typically noisy; nevertheless, plots in Fig. 3 demonstrates that the simulation has accurately captured the overall Q-factor behavior when compared to the measured data.

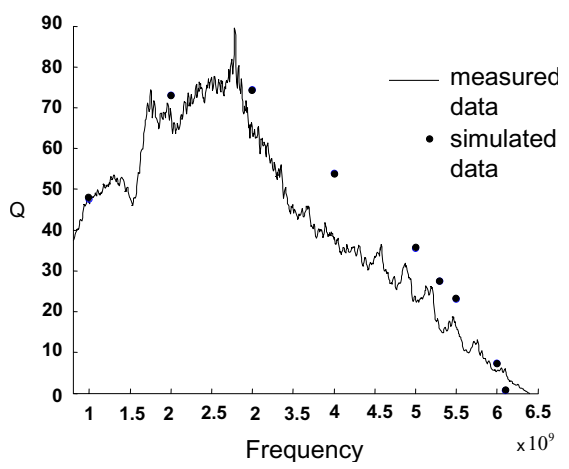

Figure 3: Measured and simulated Q-factors for the RF inductor described in Sec. 5.2.

\subsection{Substrate Effects on a Square 7-turn RF Inductor}

After establishing the validity of the solver in the previous section, this section presents the quantitative analysis of the effect of a conducting silicon substrate $\left(\rho=10 \Omega \cdot \mathrm{cm}, \varepsilon_{r}=11.7\right)$ on a large square 7-turn RF inductor in comparison to the same RF inductor simulated in free space without the substrate. The RF inductor structure is shown in Fig. 4b. For the substrate analysis, the inductor is embedded in a silicon oxide layer $\left(\varepsilon_{r}=3.9\right)$ and situated $5 \mu m$ above the substrate. It is constructed from copper wires that are $1 \mu m$ thick and $10 \mu m$ wide. The separation distance between the turns is $2 \mu \mathrm{m}$, and the overall area of the inductor is $0.25 \mathrm{~mm}^{2}$.

Fig. 5 shows that the RF inductor's performance is severely degraded in the presence of the substrate. The frequency at which self-resonance occurs is reduced from $3.5 \mathrm{GHz}$ to $0.9 \mathrm{GHz}$, indicating a decrease in the frequency range at which proper inductor operation can be ensured. The high-frequency Q-factors are reduced as well, from 23 at self-resonating frequency without the substrate to 7 with the substrate. This indicates that due to substrate effects, eddy current losses induced in the substrate may, for instance, significantly compromise the selectivity of RF filters or local oscillators.

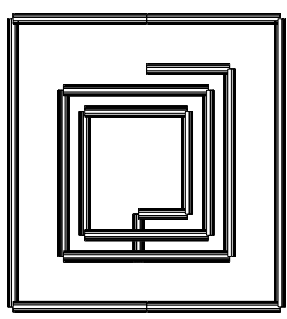

(a)

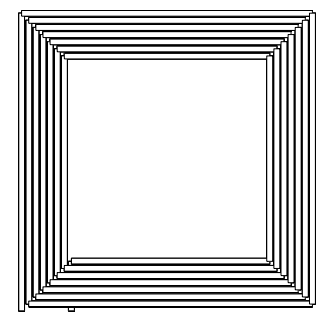

(b)
Figure 4: a. A square 2.75-turn RF inductor with ground ring; b. A square 7-turn RF inductor. 


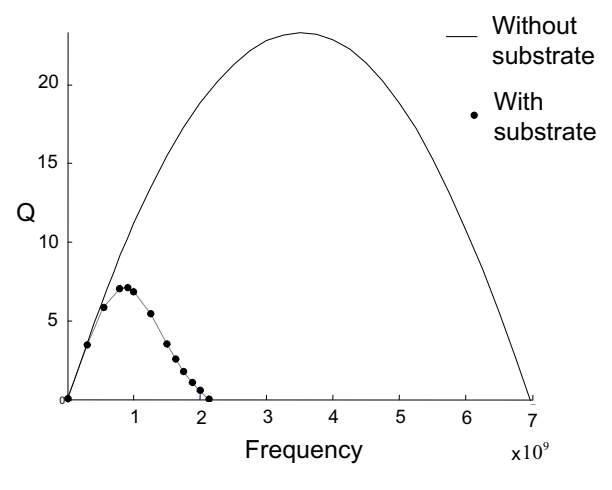

Figure 5: Q-factors with and without the substrate for the RF inductor described in Sec. 5.3.

\subsection{Full-wave Effects on a MCM Transmission Line}

A transmission line is constructed using two copper wires that are $5 \mu \mathrm{m}$ thick, $10 \mu \mathrm{m}$ wide, and $3 \mathrm{~cm}$ long. The two lines are situated $1 \mathrm{~cm}$ apart at a height $180 \mu \mathrm{m}$ above the substrate in the oxide layer. The properties of the oxide layer and the substrate are the same as the MCM in Sec. 5.2. In Fig. 6, one set of resistance results is generated in quasi-static mode where the full-wave kernels are replaced with the $\frac{1}{R}$ kernels, and the other set is generated in fullwave mode.

In Fig. 6, the full-wave effects are manifested as discrepancies in the resonance peaks between the data generated in the full-wave mode and those in quasi-static mode. These discrepancies increase with frequency, reflecting on the full-wave mode's ability, or, the quasi-static mode's inability, to capture high-frequency radiation losses in the system.

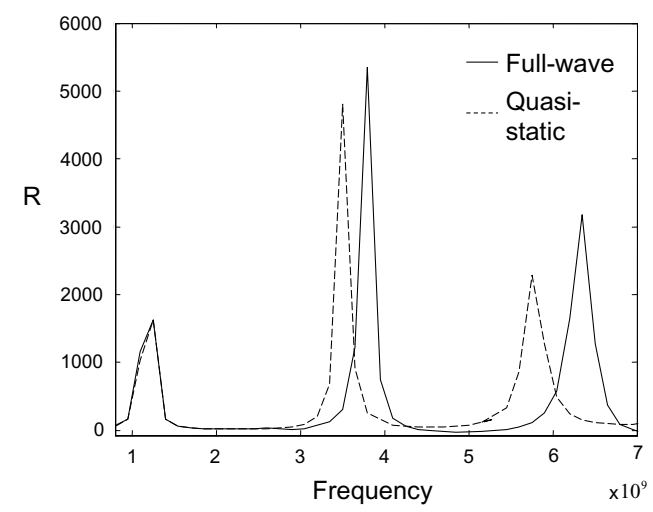

Figure 6: Resistance comparison between full-wave and quasistatic simulations on the transmission line structure in Sec. 5.4.

\section{CONCLUSION AND ACKNOWLEDGEMENTS}

This paper describes a set of novel techniques in a full-wave 3D MPIE solver that is capable of extracting conductor impedance in the presence of a layered substrate. Much of the efficiency of this solver comes from its implementation of a set of accelerated integration schemes tailored to a collection of full-wave, closed-form kernels. These kernels are derived from the application of the complex image theory, the use of which eliminates the need to explicitly discretize the substrate volume or surface. It has been shown in the paper that both the 3D filament integrations and 2D panel integrations of the complex image kernels in the solver can be reduced to a sum of 1D integrals over segments bounding either the filament volumes or panel surfaces. Examples have validated the solver's accuracy in comparison to measurement data, proved the necessity of its full-wave mode, demonstrated its flexibility in handling both package and IC level impedance analysis, and established its efficiency by achieving a $9.8 \mathrm{X}$ reduction in filament integration cost.

This work is supported in parts by the MARCO Gigascale Systems Research and Interconnect Focus Centers, the MARCO Semiconductor Research Corporation, and the DARPA Neocad Program.

\section{REFERENCES}

[1] K.A. Michalski. "Electromagnetic Scattering and Radiation by Surfaces of Arbitrary Shape in Layered Media, Part I: Theory." IEEE Trans. Antennas Propagat., Vol.38, No. 3, March 1990.

[2] J.J. Yang, Y.L. Chow, D.G. Fang. "Discrete Complex Images of a Three-dimensional Dipole Above and Within a Lossy Ground." IEE PROC-H, Vol. 138, No. 4, Aug. 1991.

[3] Y.L. Chow, J.J. Yang, and G.E. Howard. "A Closed-Form Spatial Green's Function for the Thick Microstrip Substrate." IEEE Trans. Microwave Theory Tech., Vol. 39, No. 3, March 1991.

[4] G. Dural and M.I. Aksun. "Closed-Form Green's Functions for General Sources and Stratified Media". IEEE Trans. Microwave Theory Tech., Vol. 43, No. 7, July 1995.

[5] M.I. Aksun. "A Robust Approach for the Derivation of Closed-Form Green's Functions." IEEE Trans. Microwave Theory and Tech., Vol. 44, No. 5, May 1996.

[6] N. Hojjat, S. Safavi-Naeini, R. Faraji-Dana, and Y.L. Chow. "Fast Computation of the Nonsymmetrical Components of the Green's Function for Multilayer Media using Complex Images." IEEE Trans. Antennas Propagat. Vol. 145, no.4, Aug. 1998.

[7] D. Wilton, S.M. Rao, A.W. Glisson, etc. "Potential Integrals for Uniform and Linear Source Distributions on Polygonal and Polyhedral Domains." IEEE Trans. Antennas Propagat., Vol. AP 32, no.3, March, 1984.

[8] J. Tan. "Full Wave Analysis of Transmission Lines in a Multilayer Substrate with Heavy Dielectric Losses." IEEE Trans. Components, Packaging and Manufacturing Technology, Vol. 19, No. 3, Aug. 1996

[9] A. Polycarpou. "The Finite-Element method for Modeling Circuits and Interconnects for Electronic Packaging." IEEE Trans. Microwave Theory Tech., Vol. 45, No. 10, Oct. 1997.

[10] A. Niknejad and R. Meyer. "Analysis, Design, and Optimization of Spiral Inductors and Transformers for Si RF IC's." IEEE J. Solid-State Circuits, Vol. 33, No. 10, Oct. 1998.

[11] C. Chen, T. Lee, N.Murugesan and S. Hagness. "Generalized FDTD-ADI: An Unconditionally Stable Full-Wave Maxwell's Equations Solver for VLSI Interconnect Modeling." Computer Aid Design, 2000.

[12] J. Zheng, V. Tripathi, and A. Weisshaar. "Characterization and Modeling of Multiple Coupled On-Chip Interconnects on Silicon Substrate." IEEE Trans. Microwave Theory Tech., Vol. 49, No. 10, Oct. 2001.

[13] H. Ymeri, B. Nauwelaers, K. Maex, S. Vandenberghe, and D. Roest. "New Analytic Expressions for Mutual Inductance and Resistance of Coupled Interconnects on Lossy Silicon Substrate." Si Monolithic Integrated Circuits in RF Systems, 2001

[14] J.Fan, J.L. Drewniak, H. Shi and J.L. Knighten. "DC Power-Bus Modeling and Design With a Mixed-Potential Integral-Equation Formulation and Circui Extraction." IEEE Trans. Electromagnetic Compatibility, Vol. 43 , No. 4 , Nov. 2001 .

[15] H. Lan. "A CAD-Oriented Modeling Approach of Frequency-Dependent Behavior of Substrate Noise Coupling for Mixed-Signal IC Design." Int. Symp. on Quality Electronic Design, 2003.

[16] A. Weisshaar, H. Lan, and A. Luoh. "Accurate Closed-form Expressions for the Frequency-Dependent Line Parameters of On-Chip Interconnects on Lossy Silicon Substrate." IEEE Trans. on Advanced Packaging, vol. 25, No. 2, May 2002

[17] A.E. Ruehli. "Equivalent Circuit Models for Three Dimensional Multiconductor Systems." IEEE Trans. Microwave Theory Tech., Vol. 22, March 1974.

[18] M. Kamon, N. Marques, and J. White. "FastPep: A Fast parasitic Extraction Program for Complex Three-Dimensional Geometries." Proc. of the IEEE Conference on Computer-Aided Design, San Jose, Nov. 1997.

[19] N. Marques, M. Kamon, J.K. White, and L.M. Silverira. "A mixed nodal-mesh formulation for efficient extraction and passive reduced-order modeling of 3D interconnects." Proc. of the IEEE/ACM DAC, San Francisco, CA, June 1998.

[20] J. Peters. Design of High Quality Factor: Spiral Inductors in RF MCM-D. Master's thesis, M.I.T. Sept. 2004

[21] R.F. Harrington. Field Computation by Moment Methods. MacMillan, 1968 